\title{
Does bunion surgery actually narrow the foot? Assessment of outcomes of surgery using traditional angles and a new radiographic measure of severity- the forefoot: hindfoot ratio. Correlation with clinical outcomes
}

\author{
Sarah Curran ${ }^{1 *}$, Ashok Marudanayagam² $^{2}$ Laura Beddard², Anthony Perera ${ }^{2,3,4}$
}

From 2014 College of Podiatry Conference and Exhibition

Bournemouth, UK. 13-15 November 2014

\section{Background}

Various angles have been used to grade the severity of hallux valgus deformity. They are useful in surgical planning but do not correlate with symptom severity or improvement. We feel that there is a fundamental mismatch between the width of the forefoot and the width of the hindfoot and that this is more clinically relevant, we describe two techniques for measuring this. We aim to measure the degree of foot narrowing after surgery and moreover how this correlates to the severity of preand post operative outcomes.

\section{Methods}

200 consecutive bunion operations were assessed with weight bearing radiographs. The HVA and IMA were measured according to standard practice. We also assessed forefoot width using two methods we have described. The first is the 'Forefoot Width' measured as a perpendicular to the midfoot (a technique we have previously validated). The 'Foot Ratio' is calculated as a function of the calcaneal width. Clinical outcomes were assessed using the Manchester Oxford Foot Questionnaire (MOXFQ), American Orthopaedic Foot and Ankle Surgeons (AOFAS) questionniare.

\section{Results}

Bunion surgery narrows the osseous width of the forefoot. This narrowing can be by as much as $23 \mathrm{~mm}$ in cases with severe deformity. We found that the Forefoot: Hindfoot ratio correlated with symptom severity and that normalisation of the ratio to below 2.5 was associated with better outcomes. This is important as small absolute corrections were associated with good outcomes.

\section{Conclusion}

Our measure of Forefoot Width is reproducible and allows for variations such as forefoot adductus. We feel that the Forefoot:Hindfoot ratio is more important as this determines the ability to fit into off-the-shelf footwear rather than requiring bespoke or modified footwear. This is the first study to look at the ability to narrow the forefoot and has important implications in determining patient selection and post-operative outcomes.

\section{Authors' details}

${ }^{1}$ Department of Healthcare, Cardiff Metropolitan University, Cardiff, UK.

${ }^{2}$ Cardiff and Vale University Health Board University Health Board, Cardiff, UK. ${ }^{3}$ Spire Cardiff Hospital; Cardiff, UK. ${ }^{4}$ London Foot and Ankle Centre, London, UK.

Published: 20 April 2015

\section{doi:10.1186/1757-1146-8-S1-A15}

Cite this article as: Curran et al: Does bunion surgery actually narrow the foot? Assessment of outcomes of surgery using traditional angles and a new radiographic measure of severity- the forefoot: hindfoot ratio. Correlation with clinical outcomes. Journal of Foot and Ankle Research 2015 8(Suppl 1):A15. 